\title{
Spontaneous emergence of twist and the formation of a monodomain in lyotropic chromonic liquid crystals confined to capillaries
}

\author{
Jinxin $\mathrm{Fu}^{1}$, Karthik Nayani ${ }^{1,2}$, Jung Ok Park ${ }^{1,3,4}$ and Mohan Srinivasarao ${ }^{1,2,3,5}$ \\ Having a well-aligned monodomain is critical to performing fundamental studies on liquid crystals as well as exploiting them for \\ technological applications. Unlike conventional nematics, lyotropic chromonic liquid crystals are notoriously hard to align. Here, we \\ report on the homogeneous planar alignment of Sunset Yellow in a flat rectangular capillary. Remarkably, the in-plane director aligns \\ perpendicular to the long axis of the rectangular capillary resulting in a counterintuitive configuration. We rationalize the evolution of \\ this configuration from a metastable twisted configuration by considering the coupling of the curvature of the edges of the rectangle to \\ the Frank free energy via the saddle-splay contribution. In contrast, for a square capillary, the doubly-twisted configuration is the final \\ ground state. Using a simple scaling argument, we show that the aspect ratio of the confining geometry determines the ground state. \\ NPG Asia Materials (2017) 9, e393; doi:10.1038/am.2017.84; published online 30 June 2017
}

\section{INTRODUCTION}

Lyotropic chromonic liquid crystals (LCLCs) have gained increasing attention in the last two decades as an interesting yet poorly understood class of liquid crystals. ${ }^{1-8}$ They are composed of self-assembled aggregates of plank-like molecules. ${ }^{9,10}$ The aggregation is driven by the $\pi-\pi$ interaction between the polyaromatic cores. ${ }^{11} \mathrm{~A}$ well-aligned monodomain is necessary for both fundamental studies on liquid crystals and exploiting them for technological applications. ${ }^{12-16}$ However, unlike conventional nematics, LCLCs are notoriously hard to align. ${ }^{17,18}$ In this work, we report homogeneous planar alignment of Sunset Yellow (SSY), a commonly studied LCLC, forming a monodomain under geometrical confinement.

One of the many unique properties of the LCLCs is that the energy cost of twist deformation is an order magnitude lower than splay or bend deformations. ${ }^{19,20}$ This results in a variety of interesting phenomena related to the emergence of spontaneous twist, when LCLCs are confined to a curved geometry. As an example, tactoids of disodium cromoglycate $(\text { DSCG })^{21}$ and Sunset Yellow droplets ${ }^{22}$ were observed to have twisted configurations. This is unlike conventional thermotropics confined to similar geometries. In these scenarios, the splay deformation close to the surface defects (boojums) is costly, and is relieved via a less expensive twist deformation. More recently, the reflection symmetry breaking of LCLC in a cylindrical geometry has also been reported. ${ }^{23,24}$ Here the director adopts a doubly twisted configuration even though there is a deformation-free state which corresponds to the axial configuration. The stability of the persistent doubly-twisted configuration was explained by considering the saddle-splay contribution to the free energy. It should be emphasized that, unlike chiral-nematic liquid crystals, the twisted structures of LCLCs are formed in the absence of any chiral dopants.

In this work we elucidate that, in a rectangular capillary filled with SSY, a monodomain like configuration evolves from an initially doubly twisted director configuration. This results from the coupling of the curvature of the edges of the capillary to the Frank free energy via saddle-splay elasticity. The doubly twisted region is eventually replaced by a planar monodomain. In a square capillary, we observed that the doubly-twisted director configuration is the ground state.

\section{MATERIAL AND METHODS}

Sample preparation

SSY is purchased from Sigma-Aldrich (Milwaukee, WI, USA) with a purity of $90 \%$, and further purified by dissolving it in deionized water, adding ethanol to cause the SSY to precipitate, filtering to isolate the precipitate, and then drying the powder in a vacuum oven. The purified SSY is then dissolved in deionized water to make nematic SSY solutions with different concentrations. All rectangular glass capillaries are purchased from VitroCom (Mountain Lakes, NJ, USA). Without any chemical treatment, the glass wall provides degenerate planar anchoring $\left(0^{\circ}\right.$ between the substrate and the SSY director) for SSY. The capillaries are filled with isotropic SSY solutions by capillary force, and then placed on a glass slide. The ends are sealed with epoxy glue to prevent the water evaporation.

\section{Polarized light microscopy}

The sample slide is placed on a Linkam T95-PE heat stage with a temperature control accuracy of $0.1^{\circ} \mathrm{C}$. The heat stage is mounted on the $360^{\circ}$ rotation stage

\footnotetext{
${ }^{1}$ School of Materials Science and Engineering, Georgia Institute of Technology, Atlanta, GA, USA; ${ }^{2}$ Renewable Bioproducts Institute (RBI), Georgia Institute of Technology, Atlanta, GA, USA; ${ }^{3}$ Center for Advanced Research in Optical Microscopy (CAROM), Georgia Institute of Technology, Atlanta, GA, USA; ${ }^{4}$ Center for Science and Technology of Advanced Materials and Interfaces (STAMI), Georgia Institute of Technology, Atlanta, GA, USA and ${ }^{5}$ School of Chemistry and Biochemistry, Georgia Institute of Technology, Atlanta, GA, USA Correspondence: Professor M Srinivasarao, School of Materials Science and Engineering, or School of Chemistry and Biochemistry, or Center for Advanced Research on Optical Microscopy (CAROM), Georgia Institute of Technology, 801, Ferst Drive, Atlanta, GA 30332-0295, USA.

E-mail: mohan@mse.gatech.edu

Received 23 November 2016; revised 2 March 2017; accepted 14 March 2017
} 


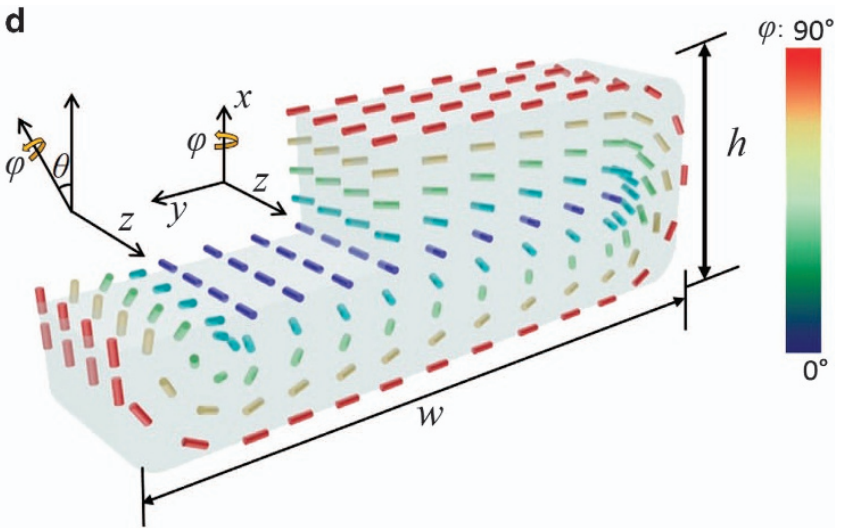

Figure 1 Crossed (a) and uncrossed ( $\mathbf{b}$ and $\mathbf{c}$ ) polarizing microscopy images of SSY with opposite twist domains in a rectangular capillary. Scale bar, $200 \mu \mathrm{m}$. (d) Schematic of the twisted director configuration in a rectangular capillary with thickness $h=20 \mu \mathrm{m}$ and width $w=200 \mu \mathrm{m}$. The color of the rods indicates the twist angle $(\varphi)$ of the local director with respect to the $z$-axis.

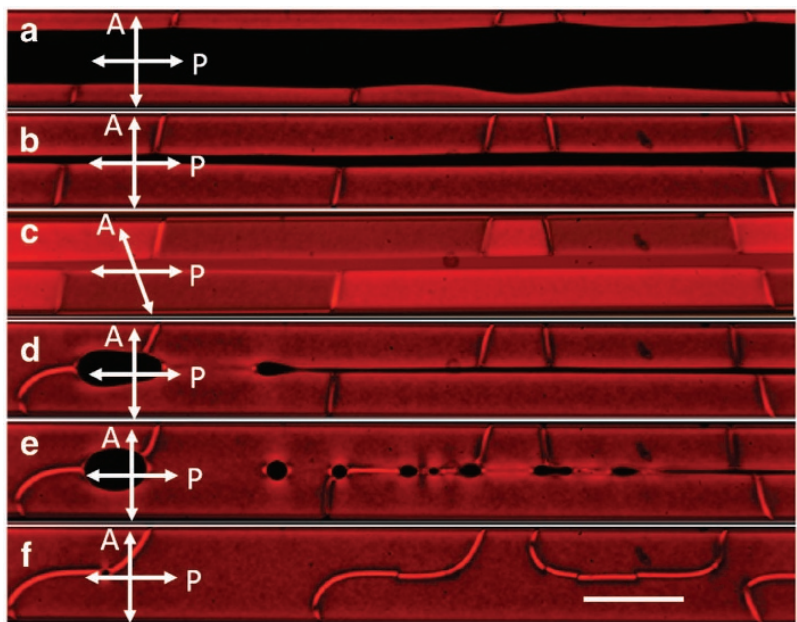

Figure 2 Time lapsed polarizing microscopy images of SSY cooling down from isotropic phase to nematic phase. The twist region of random handedness nucleates at the edges and expands to the entire capillary. Polarizer and analyzer are crossed in $\mathbf{a}, \mathbf{b}, \mathbf{d}, \mathbf{e}$ and $\mathbf{f}$, uncrossed in c. Scale bar, $200 \mu \mathrm{m}$.

of the Leica DMRX microscope. The low magnification images are obtained with a $\times 10(\mathrm{NA}=0.3)$ Leica objective and the high magnification images and fluctuation videos are obtained with $\mathrm{a} \times 100(\mathrm{NA}=1.4)$ Leica objective.

\section{RESULTS AND DISCUSSION}

Intuitively, an axial director profile with the director pointing along the long axis of the capillary is expected, when a liquid crystal is confined to a rectangular capillary with planar boundary conditions. This corresponds to a deformation free ground state. For an axial director configuration complete extinction is expected when the capillary is either parallel or perpendicular to the polarizers. This is in accordance with the expression for transmitted light intensity for the axial configuration: $I \sim \sin ^{2}(2 \alpha) \sin ^{2}\left(\frac{\delta}{2}\right)$, where $\alpha$ is the angle between the director and the polarizer and $\delta$ is the phase retardation between ordinary and extraordinary light. However, in our experiments, after cooling the SSY sample from isotropic to nematic phase, we find that there is transmitted light even when the long axis of the capillary is parallel to the polarizer as shown in Figure 1a. This hints that the director might be twisted. Furthermore, in the same figure, we can clearly see disclination lines that separate different domains. We establish that these are domains of opposite twist from the intensity profile of images obtained by uncrossing the polarizers. Figure $1 \mathrm{~b}$ and $\mathrm{c}$ correspond to images with polarizers making an angle of $70^{\circ}$ and $110^{\circ}$ with each other. As can be appreciated from Figure $1 b$ and $c$, the intensity profiles are complimentary for the two polarizer-orientations. This is an expected result if the light polarization twists in an opposite sense in the two domains. Additional proof of a twisted director profile is garnered by looking at the fluctuations of the director at different depths of the capillary, as shown in the videos and images in Supplementary information. Figure 1d is a schematic that illustrates the doubly twisted director profile that is consistent with the experimental results.

When the sample is cooled from the isotropic phase, we observe that the twist originates at the edges of the capillary. This is shown in Figure 2a. The twisted fronts approach each other upon further cooling, engulfing the isotropic region in the center as shown in Figure $2 \mathrm{~b}-\mathrm{e}$. From the image under uncrossed polarizers (Figure 2c), we see that there are domains of opposite twist at both edges, which are separated by disclination lines. The twisted domains are spatially random and have equal probability of acquiring either handedness. When the two twisted fronts meet in the center, the twisted domains of the same handedness merge; while the ones with opposite handedness are separated by disclination lines. This can be clearly seen by comparing Figure $2 \mathrm{c}$ with Figure $2 \mathrm{f}$.

We provide an explanation using scaling arguments, the preference of a doubly twisted director configuration as opposed to an axial configuration. We note that the edges of the rectangular capillary are curved as seen from the cross-section image of the capillary in Supplementary Figure $\mathrm{S} 1 .^{25}$ Hence, we make a simplistic assumption of treating the rectangular capillary as two flat planes capped by two semi-cylindrical edges, as illustrated in Figure 1d. Near the curved edges, the director would favor a doubly twisted profile. This is rationalized by the contribution of the saddle-splay term which lowers the free energy of the doubly twisted director configuration. Consider the Frank free energy expression:

$$
\begin{aligned}
F= & \frac{1}{2} \int d V\left[K_{11}(\nabla \cdot \boldsymbol{n})^{2}+K_{22}(\boldsymbol{n} \cdot \nabla \times \boldsymbol{n})^{2}+K_{33}(\boldsymbol{n} \times \nabla \times \boldsymbol{n})^{2}\right. \\
& \left.-\left(K_{24}+K_{22}\right) \nabla \cdot(\boldsymbol{n}(\nabla \cdot \boldsymbol{n})+\boldsymbol{n} \times \nabla \times \boldsymbol{n})\right]
\end{aligned}
$$

where $K_{11}, K_{22}$ and $K_{33}$ are the elastic constants associated with the well understood splay, twist and bend bulk deformations. Note that the volume integral of the saddle splay contribution can be reduced to a surface integral by using Gauss theorem. ${ }^{24}$ For planar anchoring, it is illuminating to represent the surface integral of the saddle splay contribution in terms of the principal curvatures: 

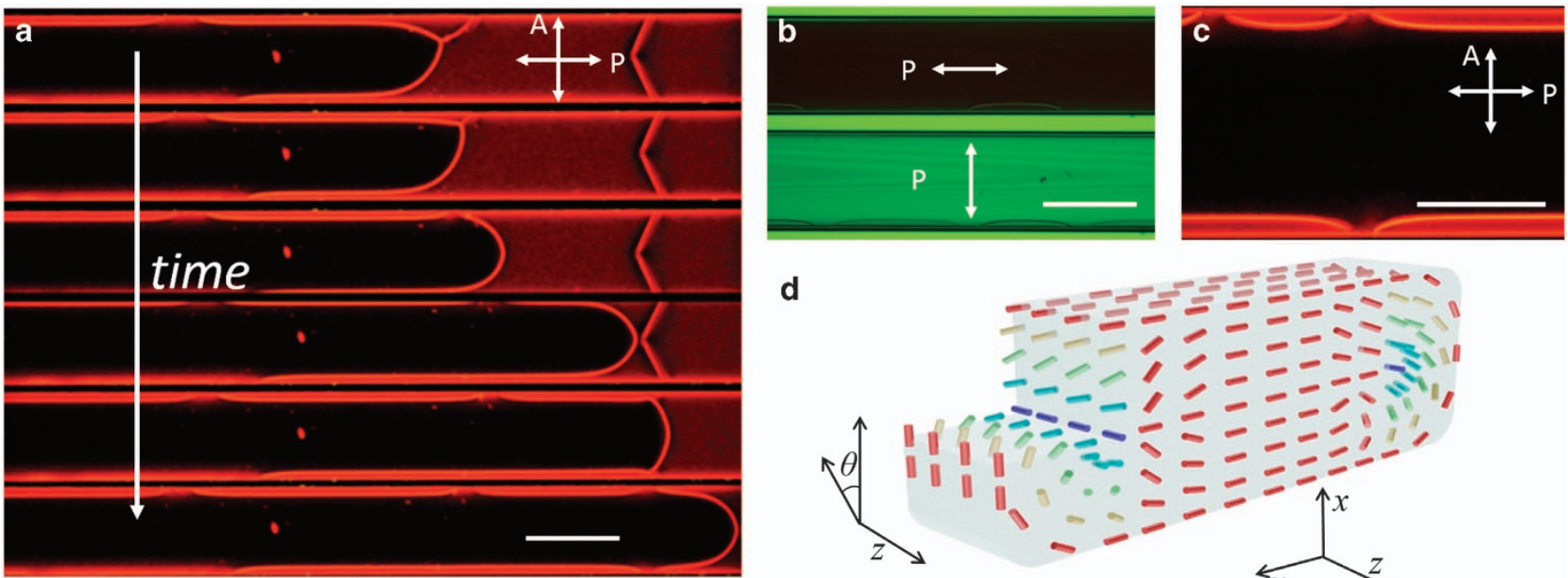

d

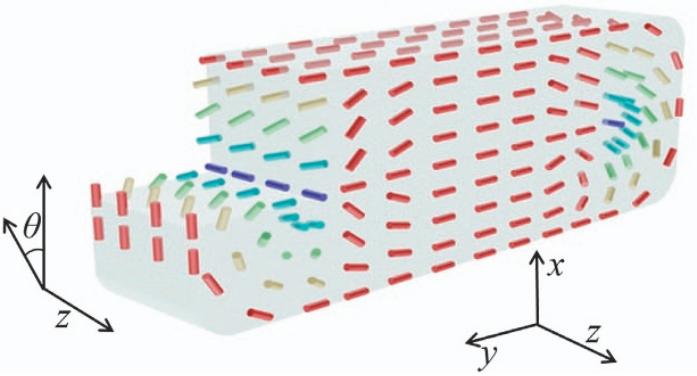

Figure 3 (a) Time evolution of the twisted configuration taken over by the monodomain like configuration. (b) Polarized light absorption of green light $(551 \mathrm{~nm}$ ) of the monodomian region. (c) Magnified crossed POM image of the monodomain region and twisted region separated by the half disclination lines. (d) Schematic of the director configuration of monodomain in center and twist near the edges. Scale bar is $200 \mu \mathrm{m}$ in $\mathbf{a}$ and $\mathbf{b}$, and $100 \mu \mathrm{m}$ in $\mathbf{c}$.

$F_{24}=-\frac{1}{2}\left(K_{24}+K_{22}\right) \int d S\left(k_{1} n_{1}^{2}+k_{2} n_{2}^{2}\right)$, where $k_{1}$ and $k_{2}$ are the principal curvatures at a point on the surface, and $n_{1}$ and $n_{2}$ the director components along the corresponding directions. Specifically, for a cylinder (or half cylinder): $n_{1}=n_{\theta}$ and $n_{2}=n_{\mathrm{z}}$; hence $k_{1}=1 / R$ and $k_{2}=0$, where $R=h / 2$ is the radius of the cylinder with $h$ being the height of the capillary, $n_{\mathrm{z}}$ is the director component along the long axis of the capillary and $n_{\theta}$ is the azimuthal component of the director, as shown in Figure 1d. Hence, the saddle splay term drives the director to point along the $n_{\theta}$ direction. For a twist angle close to $180^{\circ}$ the contribution of $K_{24}$ to the free energy per unit length would be $-\pi K_{24}$, which doesn't depend on the value of $R . .^{24}$ In the flat region, the $K_{24}$ contribution is zero.

Although it is favorable to twist at the edges, the twisting in the flat region costs energy. Imagine a simple case of a $1 \mathrm{D}$ twist of the director along the height of the capillary. The simple scaling argument would estimate the energy cost per unit length in the flat region to be of the order $K_{22} \frac{w}{h}\left(\sim 10 K_{22}\right.$, for our geometry). This can be rationalized by noting that the twist deformation is along the height of the capillary and hence the twist energy density scales as $\sim \frac{1}{h^{2}}$. Multiplying with the area of the capillary $\left(w^{\star} h\right)$ we get the scaling of the twist deformation cost per unit length to be $K_{22} \frac{w}{h}$. Hence the deformation cost of having a doubly twisted director $\left(F_{\mathrm{DT}}\right)$ in a rectangular capillary is: $F_{\mathrm{DT}} \sim\left(10 K_{22}-\pi K_{24}\right)$. Previous experimental estimates of $K_{24}$ for SSY are about the order $50 K_{22} \cdot{ }^{23,24}$ Clearly this implies, $F_{\mathrm{DT}}<0$, and the doubly twisted director is preferable when compared to axial configuration $\left(F_{\text {axial }}=0\right)$. This explains the initial doubly twisted director configuration in the rectangular capillary.

However, in a few hours the doubly twisted director configuration evolves to a configuration that forms a monodomain in the flat regions of the capillary but remains twisted at the edges. A typical transition from a metastable doubly twisted configuration to a monodomain like configuration is shown as the time-lapsed images in Figure 3a. The orientation of the director in the monodomain region can be clearly discerned from the transmission images using polarized monochromatic light $(551 \pm 4 \mathrm{~nm})$ as shown in Figure $3 \mathrm{~b}$. When the polarizer is along the long axis of the capillary, the image is dark indicating complete absorption of the incident light. In contrast, there is a strong transmission when the polarizer is along the short axis of the capillary. SSY has a broad absorption band around $400-530 \mathrm{~nm}$, resulting from $\mathrm{N}=\mathrm{N}$ and $\mathrm{N}-\mathrm{H}$ bonds in the molecular plane. ${ }^{26}$ Therefore, the director, which is perpendicular to the molecular plane, is along the short axis of the rectangular capillary. The orientation of a SSY molecule, an aggregate, and the directors are illustrated with schematics in Supplementary Figure S2. The director at the edges is still twisted and a disclination line of strength of $1 / 2$ separates it from the monodomain region. The two regions separated by the disclination line are clearly observed in the magnified image of Figure 3c. Figure $3 \mathrm{~d}$ is the schematic of the director configuration of monodomain in center and twist near the edges.

For the new monodomain configuration there is no energy cost in the flat region. However, there is an added penalty of having two halfstrength disclination lines. ${ }^{27,28}$ The energy cost of the disclination core per unit length is of the order of $\pi \mathrm{Km}^{2}{ }^{29-31}$ where $m=1 / 2$ is the strength of the line. As twist is the dominant deformation around the core, the energy cost of the core of the two lines is $\pi \frac{K_{22}}{2}$. Clearly, the cost of having two disclination lines is an order of magnitude lower than doubly twisting in the flat region $\left(\sim 10 \mathrm{~K}_{22}\right)$. The monodomain like configuration is stable for weeks and is likely the ground state for SSY in rectangular capillaries. We also observed that the monodomain structure formed in the nematic phase would be preserved when the sample is cooled down to the columnar phase, as shown in Supplementary Figure S3.

In order to further confirm our theoretical analysis, we perform the same experiments with square capillaries with widths of 50 and $100 \mu \mathrm{m}$ for comparison. From the cross-polarizers image in Figure $4 \mathrm{a}$ and the uncross-polarizers images in Figure $4 \mathrm{~b}$ and c, we can draw the same conclusion that the director of SSY liquid crystal is twisted in the square capillary as we did for the rectangular capillary. However, the doubly twisted configuration in both 50 and $100 \mu \mathrm{m}$ square capillaries keeps for months if the capillary is sealed well, and never changes to a monodomain like configuration as it does in a rectangular capillary. The difference can be well explained by our previous scaling arguments. The total free energy cost is: $F_{\mathrm{DT}} \sim\left(K_{22} \frac{w}{h}-\pi K_{24}\right)$ for a doubly-twisted configuration; and $F_{\text {Mono }} \sim\left(\pi \frac{K_{22}}{2}-\pi K_{24}\right)$ for a monodomain like configuration in a rectangular capillary. For a square capillary, the aspect ratio $\frac{w}{h}$ is 1 , causing that $F_{D T}$ is smaller than $F_{\text {Mono }}$. Therefore, a doubly-twisted configuration is more favorable to SSY liquid crystal confined in a square capillary. One can also have a better estimation of the core energy cost of the half-strength disclination line, by making a series of rectangular capillaries of different aspect ratios and checking their ground state configurations. 


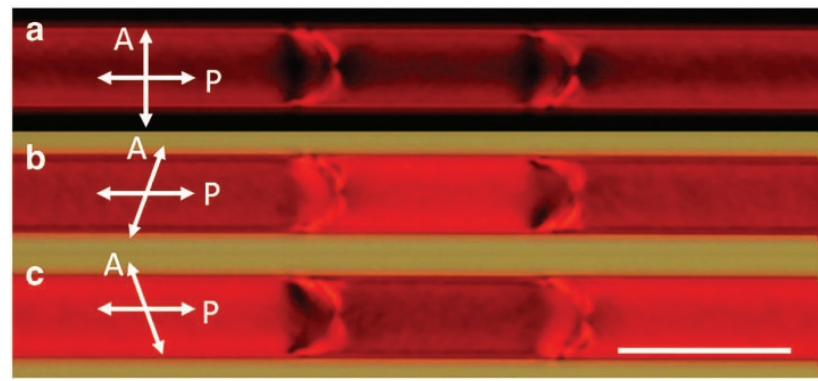

Figure 4 Crossed (a) and uncrossed ( $b$ and $\mathbf{c}$ ) polarizing microscopy images of SSY with opposite twist domains in a square capillary. Scale bar, $100 \mu \mathrm{m}$.

We also filled thermotropic liquid crystal (5CB) into the same square and rectangular capillaries. From the polarizing microscopy images and the Raman spectra shown in Supplementary Figure S4, it is seen that in both cases (square and rectangular), $5 \mathrm{CB}$ is aligned along the long axis of the capillaries. This is opposite to what is observed for SSY. The contrasting phenomenology observed for the thermotropics and LCLCs underlines the role of the extremely small twist elastic constant $K_{22}$ of the LCLCs on the global director configurations of the liquid crystals.

\section{CONCLUSION}

We observe a spontaneous doubly-twisted structure of SSY lyotropic liquid crystal in both rectangular and square capillaries. The twist structure in a flat rectangular capillary without any anchoring coating eventually evolves to a well-aligned planar mono domain, which will be very useful for lyotropic liquid crystals study like order parameters and viscoelastic constants measurements. We elucidate the key role of the saddle-splay elastic constant on the formation of the doubly twisted structure in a rectangular/square capillary, where curvature only exists at the edges. With a simple scaling argument, we explain the evolution of the monodomain like configuration from the initially doublytwisted configuration, and the different ground states of lyotropic chromonic liquid crystals under rectangular and square confinement.

\section{CONFLICT OF INTEREST}

The authors declare no conflict of interest.

\section{ACKNOWLEDGEMENTS}

KN acknowledges funding from RBI fellowship.

1 Cox, J. S. G., Woodard, G. D. \& Mccrone, W. C. Solid-state chemistry of cromolyn sodium (disodium-cromoglycate). J. Pharm. Sci. 60, 1458-1465 (1971).

2 Hartshorne, N. H. \& Woodard, G. D. Mesomorphism in the system disodium chromoglycate-water. Mol. Cryst. Liq. Cryst. 23, 343-368 (1973).

$3 \mathrm{Yu}$, L. J. \& Saupe, A. Deuteron resonance of D20 of nematic disodium-cromoglycate water-systems. Mol. Cryst. Liq. Cryst. 80, 129-134 (1982).

4 Attwood, T. K., Lydon, J. E. \& Jones, F. The chromonic phases of dyes. Liq. Cryst. 1, 499-507 (1986).

5 Collings, P. J., Dickinson, A. J. \& Smith, E. C. Molecular aggregation and chromonic liquid crystals. Liq. Cryst. 37, 701-710 (2010).

6 Masters, A. Chromonic liquid crystals: more questions than answers. Liq. Cryst. Today 25, 30-37 (2016).

7 Tam-Chang, S. W. \& Huang, L. M. Chromonic liquid crystals: properties and applications as functional materials. Chem. Commun. 17, 1957-1967 (2008).

8 Lydon, J. Chromonic liquid crystalline phases. Liq. Cryst. 38, 1663-1681 (2011).

9 Lydon, J. Chromonic review. J. Mater. Chem. 20, 10071-10099 (2010).

10 Park, H.-S. \& Lavrentovich, O. D. in Liquid Crystals Beyond Displays. 449-484 (John Wiley \& Sons, Inc., Hoboken, NJ, USA, 2012).
11 Hunter, C. A. \& Sanders, J. K. M. The nature of Pi-Pi interactions. J. Am. Chem. Soc. 112, 5525-5534 (1990).

12 Ould-Moussa, N., Blanc, C., Zamora-Ledezma, C., Lavrentovich, O. D., Smalyukh, I. I., Islam, M. F., Yodh, A. G., Maugey, M., Poulin, P., Anglaret, E. \& Nobili, M. Dispersion and orientation of single-walled carbon nanotubes in a chromonic liquid crystal. Liq. Cryst. 40, 1628-1635 (2013).

13 Park, H. S., Agarwal, A., Kotov, N. A. \& Lavrentovich, O. D. Controllable side-by-side and end-to-end assembly of Au nanorods by lyotropic chromonic materials. Langmuir 24, 13833-13837 (2008).

14 Guo, F., Mukhopadhyay, A., Sheldon, B. W. \& Hurt, R. H. Vertically aligned graphene layer arrays from chromonic liquid crystal precursors. Adv. Mater. 23, 508-513 (2011).

15 Shiyanovskii, S.V., Schneider, T., Smalyukh, I. I., Ishikawa, T., Niehaus, G. D., Doane, K. J., Woolverton, C. J. \& Lavrentovich, O. D. Real-time microbe detection based on director distortions around growing immune complexes in lyotropic chromonic liquid crystals. Phys. Rev. E 71, 020702 (2005).

16 Yao, X. X., Nayani, K., Park, J. O. \& Srinivasarao, M. Orientational order of a lyotropic chromonic liquid crystal measured by polarized raman spectroscopy. J. Phys. Chem. B 120, 4508-4512 (2016).

17 Jeong, J. W., Han, G. H., Johnson, A. T. C., Collings, P. J., Lubensky, T. C. \& Yodh, A. G. Homeotropic alignment of lyotropic chromonic liquid crystals using noncovalent interactions. Langmuir 30, 2914-2920 (2014).

18 Martens, K., Funck, T., Kempter, S., Roller, E. M., Liedl, T., Blaschke, B. M., Knecht, P., Garrido, J. A., Zhang, B. R. \& Kitzerow, H. Alignment and graphene-assisted decoration of lyotropic chromonic liquid crystals containing DNA origami nanostructures. Small 12, 1658-1666 (2016).

19 Zhou, S., Nastishin, Y. A., Omelchenko, M. M., Tortora, L., Nazarenko, V. G., Boiko, O. P. Ostapenko, T., Hu, T., Almasan, C. C., Sprunt, S. N., Gleeson, J. T. \& Lavrentovich, O. D. Elasticity of lyotropic chromonic liquid crystals probed by director reorientation in a magnetic field. Phys. Rev. Lett. 109, 037801 (2012).

20 Zhou, S., Neupane, K., Nastishin, Y. A., Baldwin, A. R., Shiyanovskii, S. V., Lavrentovich, O. D. \& Sprunt, S. Elasticity, viscosity, and orientational fluctuations of a lyotropic chromonic nematic liquid crystal disodium cromoglycate. Soft Matter 10, 6571-6581 (2014)

21 Tortora, L. \& Lavrentovich, O. D. Chiral symmetry breaking by spatial confinement in tactoidal droplets of lyotropic chromonic liquid crystals. Proc. Natl Acad. Sci. USA 108, 5163-5168 (2011).

22 Jeong, J., Davidson, Z. S., Collings, P. J., Lubensky, T. C. \& Yodh, A. G. Chiral symmetry breaking and surface faceting in chromonic liquid crystal droplets with giant elastic anisotropy. Proc. Natl Acad. Sci. USA 111, 1742-1747 (2014).

23 Davidson, Z. S., Kang, L., Jeong, J., Still, T., Collings, P. J., Lubensky, T. C. \& Yodh, A. G. Chiral structures and defects of lyotropic chromonic liquid crystals induced by saddlesplay elasticity. Phys. Rev. E 91, 050501 (2015).

24 Nayani, K., Chang, R., Fu, J. X., Ellis, P. W., Fernandez-Nieves, A., Park, J. 0. \& Srinivasarao, M. Spontaneous emergence of chirality in achiral lyotropic chromonic liquid crystals confined to cylinders. Nat. Commun. 6, 8067 (2015).

$25 \mathrm{Yao}, \mathrm{X}$. Studies on lyotropic chromonic liquid crystals in nematic and biphasic regions. (Georgia Institute of Technology, Atlanta, GA, USA, 2011).

26 Horowitz, V. R., Janowitz, L. A., Modic, A. L., Heiney, P. A. \& Collings, P. J. Aggregation behavior and chromonic liquid crystal properties of an anionic monoazo dye. Phys. Rev. E 72, 041710 (2005)

27 Yao, X., Rey, A., Park, J. \& Srinivasarao, M. Defect dynamics in monodomain formation of a lyotropic chromonic liquid crystal under confinement. Bull. Am. Phys. Soc. 56, P9.00013 (2011).

28 Shams, A., Yao, X. X., Park, J. O., Srinivasarao, M. \& Rey, A. D. Disclination elastica model of loop collision and growth in confined nematic liquid crystals. Soft Matter 11, 5455-5464 (2015).

29 de Gennes, P. G. \& Prost, J. The Physics of Liquid Crystals. Chapter 4, 2nd ed. (Clarendon Press: Oxford, UK, 1993).

30 Chandrasekhar, S. \& Ranganath, G. S. The structure and energetics of defects in liquidcrystals. Adv. Phys. 35, 507-596 (1986).

31 Chandrasekhar, S. Liquid Crystals. Chapter 3, 2nd ed. (Cambridge University Press: Cambridge, UK, 1993).

(1) This work is licensed under a Creative Commons Attribution 4.0 International License. The images or other third party material in this article are included in the article's Creative Commons license, unless indicated otherwise in the credit line; if the material is not included under the Creative Commons license, users will need to obtain permission from the license holder to reproduce the material. To view a copy of this license, visit http:// creativecommons.org/licenses/by/4.0/

(C) The Author(s) 2017

Supplementary Information accompanies the paper on the NPG Asia Materials website (http://www.nature.com/am) 